\title{
A mechanism of B cell hyperactivity in SLE
}

Researchers have identified a mechanism for B cell hyperactivity in patients with systemic lupus erythematosus (SLE). Published in Science Translational Medicine, the data show that microRNAs (miRNAs) miR-7, miR-21 and miR-22 enhance B-cell receptor signalling by suppressing the expression of PTEN, a process that could contribute to the characteristic production of autoantibodies in SLE.

Phosphatidylinositol 3,4,5-trisphosphate 3-phosphatase and dual-specificity protein phosphatase PTEN is a known tumour suppressor, but deficiency of the protein has also been shown to cause an SLE-like disease in mice.

"We relied on the mouse models to point us in the right direction," comments Peter Lipsky, corresponding author of the study. By fluorescence-activated cell sorting, his team showed that B-cell expression (except memory B cells) of PTEN was lower in patients with SLE than in healthy donors.
Importantly, the researchers also identified an inverse correlation between the level of PTEN expression and SLE disease activity.

\section{4 ...miR-7, miR-21 and}

miR-22 enhance B cell receptor signalling by suppressing the expression of PTEN... 7t

SLE B cells stimulated ex vivo with IL-21, a known inducer of PTEN, had defective responses. There was also a defect in anti-IgM and CD40L-induced Akt phosphorylation in these cells. However, IL-21-induced STAT3 and PTEN mRNA expression were not defective, indicating that the defect in the SLE B cells was not in signalling or transcription, rather, Lipsky says it "pointed toward an abnormality in miRNA regulation."

Known regulators of PTEN, miR-21 and miR-22, were therefore considered as candidates for a role in SLE B-cell activation. By TargetScan, the researchers also predicted miR-7 to be involved. All three miRNAs were found at a higher concentration in SLE B cells than in normal B cells and these miRNAs were shown to suppress PTEN expression. Finally, with an antagomir of miR-7, the team showed that miR-7 negatively regulates $P T E N$ expression in human $B$ cells and thereby identify a new candidate for therapeutic regulation.

Lipsky says of translating this ongoing research, "We are pursuing this in both mouse and human lupus to fine tune our understanding of the regulation of B cell activation and attempt to determine whether these findings provide the basis for a novel clinical intervention in lupus."

Nicholas J. Bernard

Original article $\mathrm{Wu}, \mathrm{X} . \mathrm{-N}$. et al. Defective PTEN regulation contributes to B cell hyperresponsiveness in systemic lupus erythematosus. Sci. Transl. Med. doi:10.1126/ scitransImed.3009131 